\title{
Research on the Conduction Effect of China's Corn Futures Price_Analysis Based on International Market Factors
}

\author{
Wan Zhiyi ${ }^{1, a}$ \\ ${ }^{I}$ Department of Investment, Sichuan Agricultural University, Wenjiang, Chengdu, Sichuan, China \\ a769620962@qq.com
}

\begin{abstract}
With the increasing correlation between China's corn futures and the international market, corn futures price fluctuation is closely related to the international market factors. By constructing VAR model, this paper focuses on the influence of four international trade and financial market factors -- international corn futures price, international energy price, US dollar exchange rate and corn import volume. The results of Granger causality analysis show that international energy price, US dollar exchange rate and corn import volume are the Granger reasons for the fluctuation of domestic corn futures. The impulse response analysis showed that the initial impact of the four factors was large, and then the response decreased and gradually approached the level before the impact. The results of variance decomposition analysis showed that the import volume of corn had the strongest impact on the domestic corn futures price, followed by the international energy price and the exchange rate of US dollar, while international corn futures price had the weakest impact.
\end{abstract}

Keywords: domestic corn futures price, international market factors, VAR model.

\section{我国玉米期货价格传导研究一一基于国际市场因素视 \\ 角 \\ 万志怡 ${ }^{1, a}$}

${ }^{1}$ 四川农业大学投资学系, 温江, 成都, 四川, 中国

a769620962@qq.com

\section{摘要}

随着我国玉米期货与国际市场联动性不断增强, 其价格波动与国际市场因素联系更加紧密。本文通过构建 VAR 模型，着重探讨了四个国际贸易和金融市场因素——国际玉米期货价格、国际能源价格、美元汇率、玉米进口 量波动对我国玉米期货价格波动的影响规律。Granger 因果分析结果表明，国际能源价格、美元汇率和玉米进 口量是国内玉米期货变化的 Granger 原因; 由脉冲响应分析发现国内玉米期货价格受四大因素冲击初期效应 较大, 之后响应减小, 并逐渐趋于受冲击前水平; 方差分解分析结果显示, 玉米进口量对国内玉米期货价格影 响最强，其次为国际能源价格、美元汇率，国际玉米期货价格影响相对最弱。

关键词: VAR 模型 国内玉米期货价格 国际市场因素

\section{1. 前言}

农产品期货具有价格发现以及套期保值的避险 功能，被农产品交易市场和广大农民所接受。玉米作 为三大粮食品种之一, 主要用于饲料消费、工业原料 消费以及食用消费, 其中我国饲料消费占我国玉米总
消费比超过了 $50 \%$ 。玉米也是粮食作物中用途最广、 可开发产品最多、用量最大的工业原料, 广泛用于造 纸、食品、纺织、医药等行业。据我国国家统计局数 据： 2020 年玉米产量 2.6 亿吨, 占谷物总产量的 $38.94 \%$ 。玉米市场的良好运行关乎到全国千万计的种 植户、加工企业以及下游每一位消费者的民生问题。 美国是全球最大的玉米生产和出口国, 美国政府 
将玉米生产与玉米期货联系起来, 积极鼓励和支持农 民利用期货市场进行套期保值交易，以维持玉米的价 格水平。通过玉米期货市场, 美国已成为全球玉米定 价中心，玉米期货发挥着越来越重要的作用。我国是 世界上玉米生产和消费仅次于美国的第二大国, 但玉 米期货市场相对于美国而言并不活跃。从两国产量比 值看，2020 年中国产量已经达到美国的 68.2\%; 但是 从中国玉米期货交易量与美国玉米期货交易量对比 来看， 2020 年仅占美国的 $10.8 \%$ 。

随着我国农产品金融化的发展和玉米市场开放 程度的加深, 国内玉米期货市场价格不仅受国内供求 的影响, 同时也与国际市场因素变化的关联逐渐加深, 尤其是近年来中美经贸关系恶化, 成为我国玉米期货 价格波动的重要冲击因素。为了考察国际性因素对玉 米价格的影响, 选择有代表性的国际原油价格、美元 汇率、国际玉米期货价格作为国际金融市场因素, 玉 米进口量作为国际贸易因素, 来考察国际市场因素对 国内玉米期货价格的影响。

\section{2. 相关文献评述}

我国玉米期货价格波动受到学术界的持续关注。 现有研究普遍认为国际市场因素会对国内玉米市场 产生影响。华育涛 (2021) 通过构建杜宾空间回归模 型, 证明了国际市场因素对国内玉米期货市场产生影 响。李朝鲜、付京亚 (2014) 通过研究国内玉米价格 与国际市场的动态关联, 认为国际玉米价格对国内玉 米价格影响在不断加深, 我国玉米市场对国际金融和 贸易因素的冲击均做出明显响应。杨艳涛、秦富 (2015) 通过对比分析, 认为在国际玉米贸易中, 中国玉米进 口贸易处于被动地位, 缺乏国际市场定价权和话语权。 在中美贸易战背景下, 郁纪树、刘禹峡 (2020) 指出, 中美两国玉米期货在贸易战开始后均值溢出效应有 所增强, 突出表现为中国玉米期货对美国玉米期货的 正向溢出。另外, 早在 2005 年, 司继文 (2005) 选择 最优的 Copula 函数分析我国大豆期货市场和美国日 本大豆期货市场及汇率之间的相关性结构, 阐明了期 货市场和汇率之间存在着一定的相关性。王文智 （2015）通过构建 VAR 模型, 证明了反映能源价格的 原油价格通过成本变化、替代能源寻找、市场前景等 预期对玉米期货价格产生较强的影响, 并且, 虽然汇 率在市场的自发调节下对玉米期货市场的影响弱于 能源价格, 但是仍然能够在一定程度上影响玉米期货 价格。吴海霞、葛岩等 (2018) 在研究中指出, 除了 供求基本面因素外, 原油价格、汇率在玉米市场定价 机制中也发挥着重要作用。

总体来看, 现有大部分文献仅从单个维度衡量国 际金融因素或国际贸易因素对国内玉米期货价格的 影响。目前对于能源价格、美元汇率、进口量变动如 何影响单一农产品 (如玉米) 期货价格的研究较少, 且 对此的关注一般是对数量变化的直观观察。本文研究 能源价格、美元汇率、玉米进口量和国际玉米期货价 格的变化如何影响国内玉米期货价格, 以掌握影响玉
米期货价格受影响后的波动规律, 为减小国内玉米期 货价格的剧烈波动对市场产生的不良影响提供理论 参考。

\section{3. 实证分析}

\section{1. 变量选取与数据来源}

本文以我国玉米期货价格 (CP) 作为被解释变量, 数据选取于大连商品交易所玉米期货价格; 选取芝加 哥商品交易所玉米期货价格代表国际玉米期货价格

（IP），选取纽约商业交易所原油期货价格代表国际 能源价格（CFP），并将美元对人民币汇率（ER）以及 我国玉米进口量 (IM) 作为解释变量。根据数据的可 获性和一致性原则, 本文以 2004 年 9 月至 2020 年 12 月各指标的月度数据作为样本数据。本文数据来源为 大连商品交易所官网、东方财富 Choice 金融终端、 Wind 金融终端。

\section{2 . 模型设定及检验}

为消除时间序列的异方差性，在实证检验之前， 对所有变量取自然对数, 分别用 LNCP、LNIP、LNCFP、 LNER、LNIM 表示。

\section{2. 1. 变量平稳性检验}

为避免伪回归问题, 本文采用 $\mathrm{ADF}$ 检验对所有变 量进行平稳性检验。根据检验结果, 五个变量原序列 在 $5 \%$ 的显著性水平下均不平稳, 但五个变量分别进行 一阶差分后在 $1 \%$ 显著性水平下平稳, 说明五个变量均 是一阶单整序列。

\section{表 1 变量平稳性检验结果}

\begin{tabular}{c|c|c|c|c}
\hline 变量 & $\begin{array}{c}\text { ADF 统计 } \\
\text { 量 }\end{array}$ & $\begin{array}{c}\text { 临界值 } \\
(1 \%)\end{array}$ & $\begin{array}{c}\text { 临界值 } \\
(5 \%)\end{array}$ & 结论 \\
\hline LNCP & -2.0858 & -3.4641 & -2.8763 & 不平稳 \\
\hline$\triangle$ LNCP & -9.1892 & -2.5769 & -1.9425 & 平稳 \\
\hline LNIP & -2.5088 & -3.4641 & -2.8763 & 不平稳 \\
\hline$\triangle$ LNIP & -10.4743 & -2.5769 & -1.9425 & 平稳 \\
\hline LNCFP & -0.3027 & -2.5769 & -1.9524 & 不平稳 \\
\hline$\triangle$ & -10.4574 & -2.5769 & -1.9425 & 平稳 \\
\hline LNCFP & -2.1398 & -3.4641 & -2.8763 & 不平稳 \\
\hline LNER & -7.5765 & -2.5769 & -1.9424 & 平稳 \\
\hline$\triangle$ LNER & -0.7056 & -2.5770 & -1.9425 & 不平稳 \\
\hline LNIM & -13.1749 & -2.5773 & -1.9425 & 平稳 \\
\hline$\triangle$ LNIM & \multicolumn{5}{|c}{ 注: $\triangle$ 表示序列的一阶差分 }
\end{tabular}

\subsection{2. 最优滞后阶数的确定}

构建 VAR 模型需要确定最优滞后阶数, 通常采用 LR 检验法以及 AIC、SC 准则等, 根据表 2 检验结果, 依据多数原则, 确定最优滞后阶数为 2 。然后采用 $\mathrm{AR}$ 根图示法对 VAR (2) 模型进行稳定性检验, 检验结果 
显示所有特征根倒数的模均小于 $1, \operatorname{VAR}$ (2) 模型是 平稳系统模型。

表 2 VAR 模型最优滞后阶数检验结果

\begin{tabular}{|c|c|c|c|c|c|}
\hline Lag & LR & FRE & AIC & SC & HQ \\
\hline 0 & NA & $1.27 \mathrm{e}-06$ & 0.61 & 0.70 & 0.64 \\
\hline 1 & 2222.36 & $5.86 \mathrm{e}-12$ & -11.67 & $-11.15^{*}$ & -11.46 \\
\hline 2 & $121.94^{*}$ & $3.79 \mathrm{e}-12^{*}$ & $-12.11^{*}$ & -11.14 & $-11.72^{*}$ \\
\hline 3 & 27.41 & $4.24 \mathrm{e}-12$ & -12.00 & -10.60 & -11.43 \\
\hline 4 & 15.64 & $5.07 \mathrm{e}-12$ & -11.82 & -9.98 & -11.08 \\
\hline 5 & 17.72 & $5.98 \mathrm{e}-12$ & -11.66 & -8.75 & -10.74 \\
\hline
\end{tabular}

\subsubsection{Johansen 协整检验}

由于 LNCP、LNIP、LNCFP、LNER、LNIM 五个变量 进行差分后均为一阶单整过程, 可以对其进行协整检 验。本文采用 Johansen 协整检验, 该检验方法是基 于 VAR 模型的多变量协整检验方法, 因此在构造 VAR 模型的基础上, 对变量间的关系进行协整检验, 检验 结果如下:

表 3 Johansen 协整检验结果

\begin{tabular}{|c|c|c|c|c|}
\hline $\begin{array}{c}\text { Hypothesized } \\
\text { No.of CE(S) }\end{array}$ & $\begin{array}{c}\text { Eigen } \\
\text { value }\end{array}$ & $\begin{array}{c}\text { Trace } \\
\text { Statistic }\end{array}$ & $\begin{array}{c}0.05 \\
\text { Critical } \\
\text { Value }\end{array}$ & Prob** \\
\hline None* & 0.22 & 97.78 & 69.82 & 0.0001 \\
\hline At most $1^{*}$ & 0.11 & 50.24 & 47.86 & 0.0293 \\
\hline At most 2 & 0.03 & 28.78 & 29.80 & 0.0652 \\
\hline At most 3 & 0.07 & 11.27 & 15.49 & 0.1952 \\
\hline At most 4 & 0.01 & 2.66 & 3.84 & 0.1027 \\
\hline
\end{tabular}

Johansen 协整检验结果显示, 迹统计量 97.78 大 于 $5 \%$ 的临界值 69.82 , 可以拒绝 “存在零个协整关系” 的原假设, 表明至少存在一个协整关系, 并且, 迹统 计量 50.24 大于 $5 \%$ 的临界值 47.86, 同时拒绝 “至多 存在一个协整关系” 的原假设。因此, 我国玉米期货 价格与国际市场因素之间在 $5 \%$ 的显著性水平上存在 某种长期稳定关系。

\section{2. 4. 格兰杰因果检验}

根据表 3 的结果, 在 $5 \%$ 的显著性水平下, 美元汇 率 (ER)、玉米进口量 (IM)、国际能源价格 (CFP) 的 Granger 因果检验 P 值均小于 5\%, 拒绝原假设, 表明 ER、IM、CFP 是国内玉米期货价格 (CP) 的 Granger 原因。而国际玉米期货价格 (IP) 的 Granger 因果检 验 P 值大于 $5 \%$, 不拒绝原假设, 认为 IP 不是 $\mathrm{CP}$ 的 Granger 原因。

表 4 格兰杰因果检验结果

\begin{tabular}{|c|c|c|c|}
\hline Excluded & Chi-sq & df & Prob. \\
\hline LNER & 10.82570 & 2 & 0.0045 \\
\hline LNIM & 8.713203 & 2 & 0.0128 \\
\hline LNIP & 0.806422 & 2 & 0.6682 \\
\hline LNCFP & 6.006101 & 2 & 0.0496 \\
\hline
\end{tabular}

\subsection{5.脉冲响应函数分析}

脉冲响应函数刻画的是当时间序列受到一个标 准差的外来冲击时引起内生变量的变化。本文采用脉 冲响应分析, 分别考察了国内玉米期货价格 (CP) 对 国际玉米期货价格 (IP)、进口量 (IM)、美元汇率(ER)、 国际能源价格 (CFP) 的响应路径, 以及 IP 对 $\mathrm{CP}$ 的 响应情况, 从而确定 $\mathrm{CP}$ 与各变量间的动态关系。

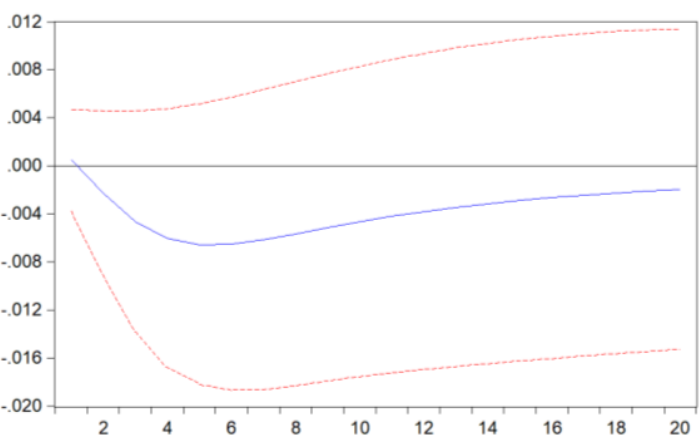

图 1 LNER 对 LNCP 的响应

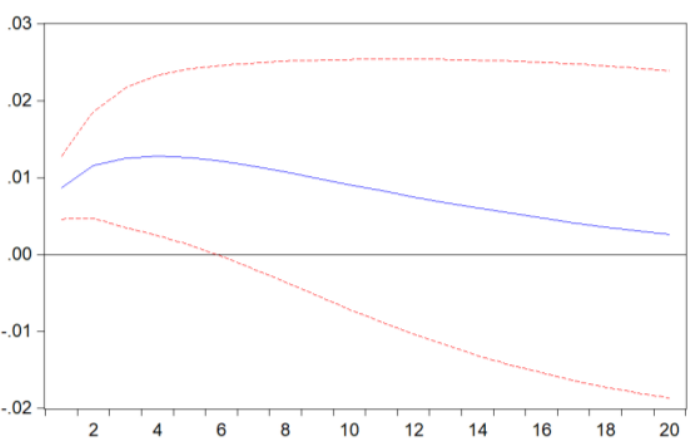

图 2 LNIP 对 LNCP 的响应

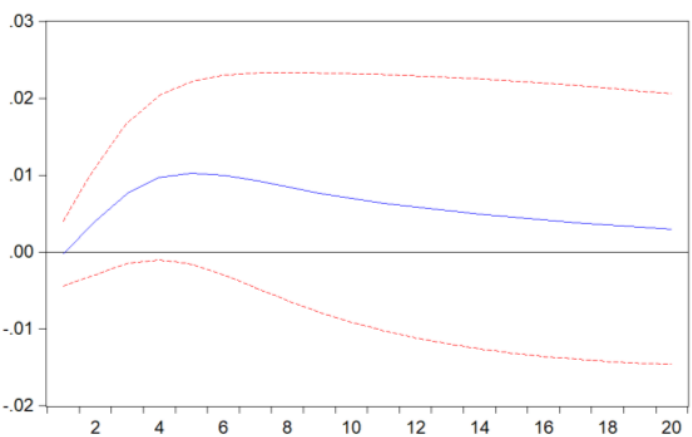

图 3 LNCFP 对 LNCP 的响应

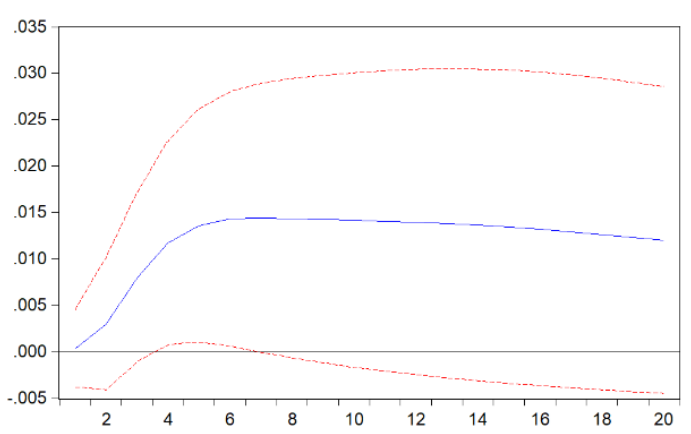

图 4 LNIM 对 LNCP 的响应 
由图 2、3、4 可看出，国际玉米期货价格、国际 能源价格和玉米进口量均对国内玉米期货价格波动 产生了正向影响。其中，国内玉米期货价格波动受国 际玉米期货价格影响较为温和, 这种影响在第 1 个月 较小，第 2 个月开始扩大，第 4 个月达到最大值后缓 慢减弱, 随后逐渐趋于平稳, 说明当期国际玉米期货 价格变动不会立即引起国内玉米价格大幅波动, 国内 玉米从下月起才开始受到较为显著的影响。国际能源 价格对国内玉米期货价格波动的影响总体平稳, 从第 1 个月开始温和上升, 到第 4 个月影响到达峰值, 随 后逐渐减弱, 表明国际能源价格对国内玉米期货价格 的影响有限。玉米进口量对国内玉米期货价格波动的 影响从第 2 个月开始逐渐显现, 在第 4 个月达到峰 值, 随后这种影响保持平稳, 这主要是因为玉米进口 从主要出口国运输到我国需要一个月左右, 因此玉米 进口导致的玉米期货价格波动存在滞后。由图 1 可看 出, 美元汇率对国内玉米期货价格波动具有负向影响 作用, 这种影响从第 1 个月开始逐渐增大, 到第 4 个 月到达峰值, 影响程度较弱, 表明当美元汇率上升时, 玉米进口成本增加, 国内玉米期货价格也随之上升, 但由于我国玉米产量较为稳定, 玉米需求主要靠国内 自给, 汇率对国内玉米期货价格影响力有限。

\section{2. 6 . 方差分解分析}

为确定各国际市场因素对我国玉米期货价格波 动影响的贡献值大小, 本文进行了方差分解分析。该 方法将系统的预测均方误差分解成系统内各变量冲 击所作贡献, 以此考察 VAR (2) 系统中任意变量冲击 的相对重要性。

根据表 5 结果, 玉米进口量、国际能源价格、美 元汇率和国际玉米期货价格的影响贡献值从第 2 期 开始出现。其中, 玉米进口量影响贡献值不断增加, 从第 2 期开始出现, 在 2-8 期迅速增加, 第 10 期达 到 8. 27\%, 并在此后保持增长。国际能源价格在 2-10 期不断增加, 在第 10 期达到最大值 $4.06 \%$ 后温和降 低。美元汇率和国际玉米期货价格对国内玉米期货价 格影响的贡献值较小, 美元汇率的贡献值在第 9 期增 长至 1.26\%后温和回落, 国际玉米期货价格贡献值虽 不断增加, 但到第 10 期的贡献值仅有 $0.46 \%$ 。从长期 来看, 玉米进口量影响贡献值相对国际能源价格、美 元汇率和国际玉米期货价格更大, 玉米进口量对国内 玉米期货价格的影响相对重要。这四者对我国玉米期 货价格影响贡献值总和达到 16\%, 其余大部分则由国 内玉米期货价格自身所解释。

表 5 方差分解分析结果

\begin{tabular}{|c|c|c|c|c|c|}
\hline Period & LNCP & LNCFP & LNER & LNIM & LNIP \\
\hline 1 & 100.000 & 0.000 & 0.000 & 0.000 & 0.000 \\
\hline 2 & 98.651 & 0.762 & 0.216 & 0.290 & 0.081 \\
\hline 3 & 95.792 & 1.911 & 0.533 & 1.599 & 0.165 \\
\hline 4 & 92.793 & 2.914 & 0.817 & 3.262 & 0.215 \\
\hline 5 & 90.411 & 3.590 & 1.023 & 4.729 & 0.247 \\
\hline 6 & 88.744 & 3.968 & 1.153 & 5.858 & 0.276 \\
\hline
\end{tabular}

\begin{tabular}{|c|c|c|c|c|c|}
\hline 7 & 87.633 & 4.135 & 1.224 & 6.698 & 0.310 \\
\hline 8 & 86.888 & 4.171 & 1.254 & 7.336 & 0.352 \\
\hline 9 & 86.364 & 4.132 & 1.257 & 7.844 & 0.465 \\
\hline 10 & 85.968 & 4.055 & 1.243 & 8.268 & 0.404 \\
\hline 11 & 85.647 & 3.960 & 1.220 & 8.638 & 0.535 \\
\hline 12 & 85.369 & 3.858 & 1.193 & 8.936 & 0.613 \\
\hline 13 & 85.119 & 3.755 & 1.164 & 9.262 & 0.793 \\
\hline 14 & 84.671 & 3.655 & 1.134 & 9.529 & 0.699 \\
\hline 15 & 84.888 & 3.559 & 1.106 & 9.771 & 0.893 \\
\hline 16 & 84.465 & 3.468 & 1.079 & 9.989 & 0.999 \\
\hline 17 & 84.269 & 3.383 & 1.054 & 10.185 & 1.109 \\
\hline 18 & 84.081 & 3.303 & 1.031 & 10.362 & 1.224 \\
\hline 19 & 83.901 & 3.228 & 1.010 & 10.520 & 1.341 \\
\hline 20 & 83.728 & 3.158 & 0.991 & 10.662 & 1.461 \\
\hline
\end{tabular}

\section{4. 结论}

\section{1. 实证分析结论}

本文通过研究 2004 年 9 月到 2020 年 12 月以来, 我国玉米期货价格波动与国际玉米期货价格、国际能 源价格、美元汇率以及玉米进口量的动态关系, 得出 如下结论: 第一, 国际贸易因素与国际金融市场因素 均能对国内玉米期货价格产生影响, 但贸易因素的影 响强度和持久性明显强于金融市场因素。这种区别可 以由脉冲响应函数反映, 玉米进口量相较于其他因素, 在受到外界冲击时, 国内玉米期货价格波动表现出更 剧烈的响应; 同时, 国内玉米期货价格变化的预测方 差 84\%左右由自身扰动引起, 由玉米进口量变化扰动 引起的预测方差是国际玉米期货价格、国际能源价格、 美元汇率总和的 2 倍, 所占比例为 $8 \%$ 。第二, 在国际 金融市场因素中，国际能源价格、美元汇率对国内玉 米期货价格波动影响强度和持久性均大于国际玉米 期货价格。脉冲响应函数分析显示, 国内玉米期货价 格受到国际能源价格和美元汇率波动冲击时响应比 受到国际玉米期货价格波动冲击时更加剧烈, 并且国 际能源价格变化量扰动引起的预测方差达到国际玉 米期货价格的 10 倍, 美元汇率达到国际玉米期货价 格的 3 倍。

此外, 影响农产品期货价格波动的国际市场因素 多且复杂，因此仅考虑国际玉米期货价格、国际能源 价格、美元汇率和玉米进口量无法完全反映国际市场 因素对我国玉米期货价格的影响, 这一点还有待进一 步研究。

\section{2. 政策建议}

经过 16 年发展, 我国玉米期货市场运行基本实 现规范稳定, 市场规模逐步扩大, 市场功能发挥水平 也逐步提高。同时，随着我国农产品市场的开放程度 不断加深以及农产品金融化的不断发展, 我国玉米期 货与国际市场的联动性不断增强, 国际贸易和金融市 场因素也成为了影响国内玉米期货市场的重要因素。 为了使我国玉米期货市场保持长足稳健的发展, 提升 玉米期货的市场功能水平，同时在波綗云论的国际格 
局中保障我国玉米期货市场的安全, 提出如下政策建 议:

\section{2. 1. 在确保粮食安全的前提下, 继续加快开 放玉米期货市场}

玉米是我国三大重要粮食作物之一, 粮食安全是 关乎经济发展、社会稳定和国家治理的重大战略问题, 尤其是在当今开放的国际市场中, 影响粮食安全的因 素越来越多，国际能源价格、美元汇率等国际金融市 场因素对玉米期货市场的影响也在逐渐加深, 因此, 确保粮食安全是玉米期货市场发展的必要前提。而作 为仅次于美国的玉米消费和生产大国, 我国玉米期货 市场不够活跃, 与美国玉米期货市场存在较大差距, 并且在国际市场中受贸易因素影响较大, 话语权不足, 需要加快开放步伐, 发挥我国玉米市场的规模优势, 加速国内玉米期货市场功能水平的提升。

\section{2. 2. 积极开拓多元化的玉米进口渠道}

尽管我国玉米产量保持平稳增长态势, 但无法完 全自给自足, 仍需依赖进口。而作为国际市场贸易因 素的玉米进口量对国内玉米期货价格能产生较大影 响, 单一的进口渠道会导致对进口来源国的过度依赖, 不利于增强我国玉米市场的竞争力, 进而影响我国玉 米期货市场的国际话语权。

\subsection{3. 健全国际金融市场的价格预警机制}

玉米作为原料广泛应用于食品消费、工业消费、 饲料消费及新型能源消费等领域, 近年来与玉米相关 的生产活动规模有增无减, 加大了对生产所需能源的 需求。当前国内玉米期货市场与国际金融市场联动性 不断增强, 以国际能源价格为代表的国际市场因素对 我国玉米期货价格产生重要影响。因此, 完善期货市 场对国际金融市场的价格预警机制能够在一定程度 上有效规避国际金融市场波动带来的风险。

\section{REFERENCES}

[1] Hua, Y.T.(2021) Analysis of price fluctuation factors in agricultural futures market -- taking corn futures as an example. J. Journal of Commercial Economics., 05: 182-184.

[2] Li, C.X., Fu, J.Y.(2014) Analysis of dynamic correlation between Chinese corn price and international market. J. Price: Theory \& Practice., 05: 61-63.

[3] Yang, Y.T., Qin, F.(2015) Correlation analysis of China's corn import trade and international market price. J. Price: Theory \& Practice., 12: 71-73.

[4] Yu, J.S., Liu, Y.T.(2020) A research on the transmission mechanism of agricultural futures price and volatility between China and the US in the context of trade war -- taking corn and soybean futures as an example. J. Times Finance., 16: 3436.

[5] Si, J.W., Meng, J.L., Gong, P.(2005) Copula analysis of correlation between domestic and foreign stock markets. J. Journal of Huazhong University of Science and Technology(Natural Science Edition)., 01: 114-116.

[6] Wang, W.Z., Wu, L.P.(2015) Research on the influence of international Factors on corn Futures price. J. Inquiry into Economic Issues., 09: 173-177.

[7] Wu, H.X., Ge, Y., Shi, H.T.(2018) Corn financialization, price formation mechanism and policy choice. J. Management Review., 30: 35-45. 